\title{
High SNR approximations of the capacity of MIMO correlated Rician channels: a large system approach.
}

\author{
Julien Dumont \\ FranceTelecom Recherche et Développement \\ Issy les Moulineaux \\ France \\ Email: dumont@crans.org
}

\author{
Walid Hachem \\ CNRS et ENST \\ LTCI, UMR 5141 \\ Paris, France \\ Email: hachem@enst.fr
}

\author{
Samson Lasaulce \\ CNRS \\ Laboratoire des Signaux et Systèmes \\ Gif sur Yvette, France \\ Email: samson.lasaulce@1ss.supelec.fr
}

\author{
Philippe Loubaton \\ Université de Marne la Vallée \\ IGM LabInfo, UMR CNRS 8049 \\ Marne la Vallée, France \\ Email: loubaton@univ-mlv.fr
}

\author{
Jamal Najim \\ CNRS et ENST \\ LTCI, UMR 5141 \\ Paris, France \\ Email: najim@enst.fr
}

\begin{abstract}
This paper studies high SNR approximations of the ergodic mutual information of block fading MIMO correlated Rician channels. The exact expression of the mutual information of such channels is quite complicated, and difficult to use to obtain convenient high SNR approximations. In this paper, it is replaced by an accurate large system approximant obtained in the case where the number of transmit and receive antennas $t$ and $r$ converge to $+\infty$ at the same rate. The large system approximant is studied at high SNR, and it is shown that 3 different behaviours are possible depending on $\frac{r}{t}$, the rank of the line of sight component and the Rician factor. The accuracy of the high SNR approximant is shown to be connected to the support of the deterministic large system approximant of the eigenvalue distribution of the Gram matrix of the channel. This allows to infer that the approximant is accurate for realistic values of $r$ and $t$ if $r \neq t$ or if $r=t$, the line of sight component is invertible and the Rician factor is greater than a certain threshold.
\end{abstract}

\section{INTRODUCTION}

The ergodic capacity of a block fading MIMO correlated Rician channel $\mathbf{H}$ is defined as the maximum of a complicated function defined on the set of positive Hermitian matrices whose normalized traces are equal to 1 . It is in particular not given by a closed form expression, and is thus difficult to analyse. Therefore, it can be useful to evaluate the capacity in certain asymptotic regimes for which it reduces to simpler terms. This paper is focused on the evaluation of the capacity when the signal to noise ratio (SNR) converges to $+\infty$. In this context, it is well established that it is optimum to transmit equal power independent symbols on the various transmit antennas. The capacity is thus reduced to the ergodic mutual information $I$ of the channel that we propose to study in the high SNR regime. For this, an obvious approach would be to use an exact expression of $I$, and then to evaluate its limit when SNR converges to $+\infty$. However, this is a difficult task because the above expressions are quite complicated; see e.g. [5] in which particular Rician channels are considered. In order to obtain interpretable results, we therefore propose to replace $I$ by its large system approximant, denoted $\bar{I}$, obtained if the number of transmit and receive antennas $t$ and $r$ converge to $+\infty$ in such a way that $\frac{r}{t} \rightarrow c$ where $0<c<+\infty$. An almost closed-form expression of $\bar{I}$ is established in [4] using random matrix methods. More precisely, it is shown that the eigenvalue distribution of matrix $\mathbf{H}^{H} \mathbf{H}$ can be approximated by a deterministic measure whose Stieljès transform is characterized in [4]. $\bar{I}$ is obtained by using that $I$ is the mathematical expectation of a particular functional of the eigenvalue distribution of the Gram matrix of the channel. Note that $\bar{I}$ has been obtained independently in [7] using the useful but non rigorous replica method. In this paper, we study the behaviour of $\bar{I}$ for fixed values of $r$ and $t$ when $S N R$ converges to $+\infty$. We show that the high SNR approximant of $\bar{I}$ has, depending on the context, three possible different expressions. It is of course important to verify if the high SNR approximant of $\bar{I}$ is a reliable estimate of $I$ for realistic values of $r, t$, and SNR. This important question is not completely theoretically solved in this paper. However, we provide some arguments which strongly suggest that the approximant is reliable if 0 does not belong to the support of the deterministic equivalent of the eigenvalue distribution of $\mathbf{H}^{H} \mathbf{H}$ (if $r \geq t$, otherwise $\mathbf{H} \mathbf{H}^{H}$ has to be considered). This allows to identify the contexts in which the approximant is relevant. We finally provide numerical experiments that sustain our claims. Engineering implications of our results will be discussed elsewhere due to the lack of space.

\section{CHANNEL MODEL}

We consider a wireless MIMO link with $t$ transmit antennas and $r$ receive antennas. In our analysis the channel matrix can possibly vary from symbol vector (or space-time codeword) to symbol vector (or space-time codeword). The channel matrix is assumed to be perfectly known at the receiver whereas the transmitter has only access to the statistics of the channel. The 
received signal can be written as

$$
\mathbf{y}(\tau)=\mathbf{H}(\tau) \mathbf{x}(\tau)+\mathbf{z}(\tau)
$$

where $\mathbf{x}(\tau)$ is the vector of transmitted symbols at time $\tau$, $\mathbf{H}(\tau)$ is the channel matrix and $\mathbf{z}(\tau)$ is a complex white Gaussian noise distributed as $\mathcal{N}\left(0, \sigma^{2} \mathbf{I}_{r}\right)$. For simplicity we will omit the time index $\tau$ from our notations. The channel input is subject to a power constraint $\operatorname{Tr}\left[\mathbb{E}\left(\mathbf{x x}^{H}\right)\right]=t$. The $r \times t$ channel matrix $\mathbf{H}$ has the following structure

$$
\mathbf{H}=\sqrt{\frac{K}{K+1}} \mathbf{A}+\frac{1}{\sqrt{K+1}} \mathbf{V}
$$

The matrix $\mathbf{A}$ is deterministic and satisfies $\frac{1}{r} \operatorname{Tr}\left(\mathbf{A} \mathbf{A}^{H}\right)=1$. $\mathbf{V}$ is a random matrix given by

$$
\mathbf{V}=\frac{1}{\sqrt{t}} \mathbf{C}^{1 / 2} \mathbf{W} \tilde{\mathbf{C}}^{1 / 2}
$$

where $\mathbf{W}$ is a zero mean independent and identically distributed complex Gaussian matrix in the sense that the real and imaginary parts of its entries are independent and have the same variance $\frac{1}{2}$. The matrices $\tilde{\mathbf{C}}>0$ and $\mathbf{C}>0$ account for the transmit and receive antenna correlation effects respectively, and satisfy $\frac{1}{t} \operatorname{Tr}(\tilde{\mathbf{C}})=1$ and $\frac{1}{r} \operatorname{Tr}(\mathbf{C})=1$ respectively. This correlation structure is often referred to as a separable or Kronecker correlation model. $K \geq 0$ is the so-called Rician factor which expresses the relative strength of the direct and scattered components of the received signal. Due to the above various normalization constraints, the signal to noise ratio is equal to $\frac{1}{\sigma^{2}}$.

The ergordic mutual information (EMI) at noise level $\sigma^{2}$ is defined by:

$$
I\left(\sigma^{2}\right)=\mathbb{E}_{\boldsymbol{H}}\left[\log \operatorname{det}\left(\mathbf{I}_{t}+\frac{1}{\sigma^{2}} \mathbf{H}^{H} \mathbf{H}\right)\right] .
$$

It is possible to obtain the analytical expression of $I\left(\sigma^{2}\right)$ (see e.g. [5] in the i.i.d. Rayleigh part case). However, it is quite complicated, and in practice very difficult to use. It is therefore useful to study $I\left(\sigma^{2}\right)$ in certain asymptotic regimes. From now on, we study $I\left(\sigma^{2}\right)$ when $\sigma^{2} \rightarrow 0$, and assume without restrictions that $r \geq t$. It is reasonnable to conjecture that

$$
I\left(\sigma^{2}\right)=t \log \frac{1}{\sigma^{2}}+\mathbb{E}_{\boldsymbol{H}}\left[\log \operatorname{det}\left(\mathbf{H}^{H} \mathbf{H}\right)\right]+\epsilon\left(\sigma^{2}\right)
$$

where $\epsilon\left(\sigma^{2}\right)$ converges to 0 if $\sigma^{2} \rightarrow 0$. It is again possible to evaluate $\mathbb{E}_{\boldsymbol{H}}\left[\log \operatorname{det}\left(\mathbf{H}^{H} \mathbf{H}\right)\right]$ in closed form, but the corresponding expression remains complicated except in particular cases. This is the reason for which we propose to replace in the following $I\left(\sigma^{2}\right)$ be its large system approximant obtained when $r$ and $t$ converge to $+\infty$ at the same rate.

\section{BACKGROUND ON THE LARGE SYSTEM BEHAVIOR OF THE ERGODIC MUTUAL INFORMATION.}

In this section, we review some of the results of [4] and specify the convergence rate of the approximant of $I\left(\sigma^{2}\right)$. In this section, parameter $\sigma^{2}$ is fixed and $r \rightarrow+\infty, t \rightarrow+\infty$ in such a way that $\frac{r}{t} \rightarrow c$. In the following, $t \rightarrow+\infty$ should be understood as $r \rightarrow+\infty, t \rightarrow+\infty, \frac{r}{t} \rightarrow c$ in order to short the notations.

Theorem 3.1: Assume that $\sup _{t}\|\mathbf{A}\|<+\infty, \sup _{t}\|\mathbf{C}\|<$ $+\infty$ and $\sup _{t}\|\tilde{\mathbf{C}}\|<+\infty$ where $\|$.$\| stands for the spectral$ norm. Then, for each $\sigma^{2}>0$, it exists two unique strictly positive terms $\delta\left(\sigma^{2}\right)$ and $\tilde{\delta}\left(\sigma^{2}\right)$ satisfying

$$
\begin{aligned}
& \delta\left(\sigma^{2}\right)=\frac{1}{t} \operatorname{Tr} \mathbf{C T}\left(\sigma^{2}\right) \\
& \tilde{\delta}\left(\sigma^{2}\right)=\frac{1}{t} \operatorname{Tr} \tilde{\mathbf{C}} \tilde{\mathbf{T}}\left(\sigma^{2}\right)
\end{aligned}
$$

where $\mathbf{T}\left(\sigma^{2}\right)$ and $\tilde{\mathbf{T}}\left(\sigma^{2}\right)$ depend on $\delta\left(\sigma^{2}\right)$ and $\tilde{\delta}\left(\sigma^{2}\right)$, and are defined by

$$
\begin{aligned}
\mathbf{T}\left(\sigma^{2}\right) & =\left[\sigma^{2}\left(\mathbf{I}+\frac{\tilde{\delta}\left(\sigma^{2}\right)}{K+1} \mathbf{C}\right)+\frac{K}{K+1} \mathbf{A}\left(\mathbf{I}+\frac{\delta\left(\sigma^{2}\right)}{K+1} \tilde{\mathbf{C}}\right)^{-1} \mathbf{A}^{H}\right]^{-1} \\
\tilde{\mathbf{T}}\left(\sigma^{2}\right) & =\left[\sigma^{2}\left(\mathbf{I}+\frac{\delta\left(\sigma^{2}\right)}{K+1} \tilde{\mathbf{C}}\right)+\frac{K}{K+1} \mathbf{A}^{H}\left(\mathbf{I}+\frac{\tilde{\delta}\left(\sigma^{2}\right)}{K+1} \mathbf{C}\right)^{-1} \mathbf{A}\right]^{-1}
\end{aligned}
$$

Function $\sigma^{2} \rightarrow \frac{1}{t} \operatorname{Tr} \tilde{\mathbf{T}}\left(\sigma^{2}\right)$ is the Stieljès transforms of a probability measure $\tilde{\mu}$ carried by $\mathbb{R}^{+}$, i.e.

$$
\frac{1}{t} \operatorname{Tr} \tilde{\mathbf{T}}\left(\sigma^{2}\right)=\int_{\mathbb{R}^{+}} \frac{1}{\lambda+\sigma^{2}} d \tilde{\mu}\left(\sigma^{2}\right)
$$

and $\tilde{\mu}$ satisfies:

$$
\frac{1}{t} \sum_{j=1}^{t} \phi\left(\lambda_{j}\right)-\int_{\mathbb{R}^{+}} \phi(\lambda) d \tilde{\mu}(\lambda) \rightarrow 0
$$

when $t \rightarrow+\infty$, where the convergence is the almost sure convergence. Here, $\left(\lambda_{j}\right)_{j=1, \ldots, t}$ represent the (random) eigenvalues of the positive Gram matrix $\mathbf{H}^{H} \mathbf{H}$, and $\phi$ is any lower bounded continuous function ${ }^{1}$. Moreover, functions $\sigma^{2} \rightarrow \frac{t}{r} \delta\left(\sigma^{2}\right)$ and $\sigma^{2} \rightarrow \tilde{\delta}\left(\sigma^{2}\right)$ are also Stieljès transforms of probability measures carried by $\mathbb{R}^{+}$. Finally, when $t \rightarrow+\infty$

$$
I\left(\sigma^{2}\right)=\bar{I}\left(\sigma^{2}\right)+O\left(\frac{1}{t}\right)
$$

where the asymptotic approximant $\bar{I}\left(\sigma^{2}\right)$ is given by

$$
\begin{gathered}
\bar{I}\left(\sigma^{2}\right)=\log \operatorname{det}\left[\mathbf{I}_{t}+\frac{\delta\left(\sigma^{2}\right)}{K+1} \tilde{\mathbf{C}}+\frac{1}{\sigma^{2}} \frac{K}{K+1} \mathbf{A}^{H}\left(\mathbf{I}_{r}+\frac{\tilde{\delta}\left(\sigma^{2}\right)}{K+1} \mathbf{C}\right)^{-1} \mathbf{A}\right] \\
+\log \operatorname{det}\left[\mathbf{I}_{r}+\frac{\tilde{\delta}\left(\sigma^{2}\right)}{K+1} \mathbf{C}\right]-t \sigma^{2} \frac{1}{K+1} \delta\left(\sigma^{2}\right) \tilde{\delta}\left(\sigma^{2}\right)
\end{gathered}
$$

Measure $\tilde{\mu}$ is called in the following the deterministic equivalent of the eigenvalue distribution of matrix $\mathbf{H}^{H} \mathbf{H}$. Remark that both $I\left(\sigma^{2}\right)$ and $\bar{I}\left(\sigma^{2}\right)$ increase linearly with $t$. (10) thus implies that the relative error $\frac{I\left(\sigma^{2}\right)-\bar{I}\left(\sigma^{2}\right)}{I\left(\sigma^{2}\right)}=O\left(\frac{1}{t^{2}}\right)$. This very fast convergence rate tends to explain why the asymptotic evaluations of the ergodic mutual information are reliable even for quite moderate numbers of antennas. Similar results have also been obtained in more restrictive contexts by using the replica method. We finally note that the term $O\left(\frac{1}{t}\right)$ in (10) depends on $\sigma^{2}$, and that it may converge to $+\infty$ when $\sigma^{2} \rightarrow 0$. This is the reason for which it is not obvious that $\bar{I}\left(\sigma^{2}\right)$ remains a reliable approximant for moderate values of $t$ and $r$ at high SNR. See the discussion below.

\footnotetext{
${ }^{1}$ In principle, (9) should be valid for bounded continuous function. As $\mathbf{H}$ is Gaussian, it can be shown that the largest eigenvalue of $\mathbf{H}^{H} \mathbf{H}$ is almost surely uniformly upper bounded; the behavior of $\phi$ when $\lambda \rightarrow+\infty$ has therefore no impact.
} 


\section{BehaViour of $\bar{I}\left(\sigma^{2}\right)$ IF $\sigma^{2} \rightarrow 0$.}

We now study $\bar{I}$ at high SNR $\sigma^{2} \rightarrow 0 . r$ and $t$ are fixed in this section. We define function $\bar{L}\left(\sigma^{2}\right)$ by

$$
\bar{L}\left(\sigma^{2}\right)=\bar{I}\left(\sigma^{2}\right)-t \log \frac{1}{\sigma^{2}}
$$

and show below that $\bar{L}\left(\sigma^{2}\right)$ is defined at $\sigma^{2}=0$, and that

$$
\bar{L}\left(\sigma^{2}\right)=\bar{L}(0)+\epsilon\left(\sigma^{2}\right)
$$

where $\epsilon\left(\sigma^{2}\right)$ converges towards 0 when $\sigma^{2} \rightarrow 0$. We now evaluate $\bar{L}(0)$. Various cases have to be considered, and we first consider the case $r=t$.

\section{A. The case $r=t$.}

If matrix $\mathbf{A}$ is invertible, we define $K_{c}$ as

$$
K_{c}=\frac{1}{t} \operatorname{Tr}_{\mathbf{C A}}{ }^{-H} \tilde{\mathbf{C}} \mathbf{A}^{-1}
$$

Proposition 4.1: If $\mathbf{A}$ is invertible and $K>K_{c}$, then $\lim _{\sigma^{2} \rightarrow 0} \delta\left(\sigma^{2}\right)=\delta_{*}$ and $\lim _{\sigma^{2} \rightarrow 0} \tilde{\delta}\left(\sigma^{2}\right)=\tilde{\delta}_{*}$ are both finite. Moreover, $\bar{L}\left(\sigma^{2}\right)$ can be written as (13) where

$$
\bar{L}(0)=\log \operatorname{det} \frac{K}{K+1} \mathbf{A} \mathbf{A}^{H}
$$

If $\mathbf{A}$ is non invertible, or if $\mathbf{A}$ is invertible and $K<K_{c}$, then $\lim _{\sigma^{2} \rightarrow+\infty} \sigma \delta\left(\sigma^{2}\right)=\xi_{*}$ and $\lim _{\sigma^{2} \rightarrow+\infty} \sigma \tilde{\delta}\left(\sigma^{2}\right)=\tilde{\xi}_{*}$ exist and are finite. The product $v_{*}=\xi_{*} \tilde{\xi}_{*}$ is the unique solution of the equation

$$
\frac{1}{t} \operatorname{Tr}\left[\frac{v}{K+1}+K \mathbf{A}^{H} \mathbf{C}^{-1} \mathbf{A} \tilde{\mathbf{C}}^{-1}\right]^{-1}=1
$$

and $\bar{L}\left(\sigma^{2}\right)$ is given by (13) where

$$
\bar{L}(0)=\begin{gathered}
\log \operatorname{det} \mathbf{C}+\log \operatorname{det} \tilde{\mathbf{C}}-t \frac{v_{*}}{K+1}+ \\
\log \operatorname{det}\left[\frac{v_{*}}{(K+1)^{2}} \mathbf{I}+\frac{K}{K+1} \tilde{\mathbf{C}}^{-1 / 2} \mathbf{A}^{H} \mathbf{C}^{-1} \mathbf{A} \tilde{\mathbf{C}}^{-1 / 2}\right]
\end{gathered}
$$

Sketch of proof. We first consider the case $\mathbf{A}$ invertible and $K>K_{c}$. Equation (13) and (15) are a matter of routine as soon as it is established that $\delta$ and $\tilde{\delta}$ converge to finite limits when $\sigma^{2} \rightarrow 0$. We therefore only justify this point. $\delta\left(\sigma^{2}\right)$ and $\tilde{\delta}\left(\sigma^{2}\right)$ are the unique strictly positive solutions of the system of equations

$$
\begin{aligned}
& \kappa-f\left(\kappa, \tilde{\kappa}, \sigma^{2}\right)=0 \\
& \tilde{\kappa}-\tilde{f}\left(\kappa, \tilde{\kappa}, \sigma^{2}\right)=0
\end{aligned}
$$

where the definition of functions $f$ and $\tilde{f}$ are obvious. It is easy to check that condition $K>K_{c}$ implies that (18) has unique strictly positive solutions $\left(\delta_{*}, \tilde{\delta}_{*}\right)$ for $\sigma^{2}=0$. Indeed, the first equation of (18) gives

$$
\delta_{*}=\frac{\delta_{*}}{K} \frac{1}{t} \operatorname{Tr} \mathbf{A}^{-H} \tilde{\mathbf{C}} \mathbf{A}^{-1} \mathbf{C}^{-1}+\frac{K+1}{K} \frac{1}{t} \operatorname{Tr}\left(\mathbf{A} \mathbf{A}^{H}\right)^{-1}
$$

As $K>K_{c}=\frac{1}{t} \operatorname{Tr} \mathbf{A}^{-H} \tilde{\mathbf{C}} \mathbf{A}^{-1} \mathbf{C}^{-1}$, this equation has a unique positive solution $\delta_{*}$. The study of the second equation gives a similar result concerning $\tilde{\delta}_{*}$. We now establish that $\delta_{*}$ and $\tilde{\delta}_{*}$ coincide with the limits of $\delta\left(\sigma^{2}\right)$ and $\tilde{\delta}\left(\sigma^{2}\right)$ when $\sigma^{2} \rightarrow 0$. It is easy to check that functions $f$ and $\tilde{f}$ can be extended in a neighbourhood of $\left(\delta_{*}, \tilde{\delta}_{*}, 0\right)$ to an holomorphic function of $(\kappa, \tilde{\kappa}, z)$ (i.e. the positive variables $\left(\kappa, \tilde{\kappa}, \sigma^{2}\right)$ can be replaced by complex variables) The last step of the proof is based on a complex version of the local inversion theorem (see e.g. [2], Proposition 6.1, p. 138). We denote by $\mathbf{J}\left(\delta_{*}, \tilde{\delta}_{*}, 0\right)$ the $2 \times 2$ Jacobian matrix of system $(18)$ at point $\left(\delta_{*}, \tilde{\delta}_{*}, 0\right)$ :

$$
\mathbf{J}\left(\delta_{*}, \tilde{\delta}_{*}, 0\right)=\left(\begin{array}{cc}
1-\left(\frac{\partial f}{\partial \kappa}\right)_{\left(\delta_{*}, \tilde{\delta}_{*}, 0\right)} & \left(\frac{\partial f}{\partial \tilde{\kappa}}\right)_{\left(\delta_{*}, \tilde{\delta}_{*}, 0\right)} \\
\left(\frac{\partial \tilde{f}}{\partial \kappa}\right)_{\left(\delta_{*}, \tilde{\delta}_{*}, 0\right)} & 1-\left(\frac{\partial f}{\partial \tilde{\kappa}}\right)_{\left(\delta_{*}, \tilde{\delta}_{*}, 0\right)}
\end{array}\right)
$$

It is easily seen that $\operatorname{det} \mathbf{J}\left(\delta_{*}, \tilde{\delta}_{*}, 0\right) \neq 0$. Therefore, there exist unique holomorphic functions of variable $z$, denoted $(\kappa(z), \tilde{\kappa}(z))$, defined in a neighborhood of 0 , and satisfying (18). For $z=\sigma^{2}>0$, functions $\kappa$ and $\tilde{\kappa}$ coincide with functions $\delta$ and $\tilde{\delta}$ because the later functions are continuously differentiable (they are Stieljès transforms of finite positive measures) and verify (18). This in particular implies that $\lim _{\sigma^{2} \rightarrow 0} \delta\left(\sigma^{2}\right)=\delta_{*}$ and $\lim _{\sigma^{2} \rightarrow 0} \tilde{\delta}\left(\sigma^{2}\right)=\tilde{\delta}_{*}$.

We now consider the case $\mathbf{A}$ non invertible or $\mathbf{A}$ invertible and $K<K_{c}$, and only check the statement related to the behavior of $\delta$ and $\tilde{\delta}$ around 0 . We denote by $\xi(\sigma)$ and $\tilde{\xi}(\sigma)$ the functions $\xi(\sigma)=\sigma \delta\left(\sigma^{2}\right)$ and $\tilde{\xi}(\sigma)=\sigma \tilde{\delta}\left(\sigma^{2}\right)$. It is easily seen that $\xi(\sigma)$ and $\tilde{\xi}(\sigma)$ are the unique positive solutions of the equations

$$
\begin{aligned}
& g(\kappa, \tilde{\kappa}, \sigma)=1 \\
& \tilde{g}(\kappa, \tilde{\kappa}, \sigma)=1
\end{aligned}
$$

where the expression of functions $g$ and $\tilde{g}$ are easy to obtain. Denote by $\phi(v)$ the function

$$
\phi(v)=\frac{1}{t} \operatorname{Tr}\left[\frac{v}{K+1}+K \mathbf{A}^{H} \mathbf{C}^{-1} \mathbf{A} \tilde{\mathbf{C}}^{-1}\right]^{-1}
$$

It is easily checked that $g(\kappa, \tilde{\kappa}, 0)=\tilde{g}(\kappa, \tilde{\kappa}, 0)=\phi(\kappa \tilde{\kappa})$. In contrast with the context $K>K_{c}$, the equations (20) reduce to the single equation $\phi(\kappa \tilde{\kappa})=1$ at $\sigma=0$. Therefore, more work is needed to prove the Proposition. It is easy to check that it exist functions $h(\kappa, \tilde{\kappa}, \sigma)$ and $\tilde{h}(\kappa, \tilde{\kappa}, \sigma)$ such that

$$
\begin{aligned}
& g(\kappa, \tilde{\kappa}, \sigma)=\phi(\kappa \tilde{\kappa})+\sigma h(\kappa, \tilde{\kappa}, \sigma) \\
& \tilde{g}(\kappa, \tilde{\kappa}, \sigma)=\phi(\kappa \tilde{\kappa})+\sigma \tilde{h}(\kappa, \tilde{\kappa}, \sigma)
\end{aligned}
$$

We omit to give the (cumbersome) analytical expression of $h$ and $\tilde{h}$. (20) can thus be written as

$$
\begin{gathered}
\phi(\kappa \tilde{\kappa})+\sigma h(\kappa, \tilde{\kappa}, \sigma)=1 \\
h(\kappa, \tilde{\kappa}, \sigma)-\tilde{h}(\kappa, \tilde{\kappa}, \sigma)=0
\end{gathered}
$$

This time, it can be shown that at $\sigma=0$, equations (22) have a unique pair of strictly positive solutions $\left(\xi_{*}, \tilde{\xi}_{*}\right)$ and that the corresponding Jacobian matrix at $\left(\xi_{*}, \tilde{\xi}_{*}, 0\right)$ is invertible. Using again the local inversion theorem, we show that $\lim _{\sigma^{2} \rightarrow 0} \xi(\sigma)=\xi_{*}$ and $\lim _{\sigma^{2} \rightarrow 0} \tilde{\xi}(\sigma)=\tilde{\xi}_{*}$.

\section{B. The case $r>t$.}

We consider now the case $r>t$, and omit the proof of the following result. 
Proposition 4.2: $\gamma_{*}=\lim _{\sigma^{2} \rightarrow 0} \sigma^{2} \delta\left(\sigma^{2}\right)$ is strictly positive and $\tilde{\delta}_{*}=\lim _{\sigma^{2} \rightarrow 0} \tilde{\delta}\left(\sigma^{2}\right)$ is finite. Moreover, $\left(\gamma_{*}, \delta_{*}\right)$ are the unique strictly positive solutions of the equations

$$
\begin{array}{lll}
\gamma_{*} & = & \frac{1}{t} \operatorname{Tr}\left[\mathbf{C}\left(\mathbf{I}+\frac{\tilde{\delta}_{*}}{K+1} \mathbf{C}+\frac{K}{\gamma_{*}} \mathbf{A} \tilde{\mathbf{C}}^{-1} \mathbf{A}^{H}\right)^{-1}\right] \\
\tilde{\delta}_{*} & =\frac{1}{t} \operatorname{Tr}\left[\tilde{\mathbf{C}}\left(\frac{\gamma_{*}}{K+1} \tilde{\mathbf{C}}+\frac{K}{K+1} \mathbf{A}^{H}\left(\mathbf{I}+\frac{\tilde{\delta}_{*}}{K+1} \mathbf{C}\right)^{-1} \mathbf{A}\right)^{-1}\right]
\end{array}
$$

and $\bar{L}\left(\sigma^{2}\right)$ is given by (13) where

$$
\bar{L}(0)=\begin{gathered}
\log \operatorname{det}\left(\mathbf{I}+\frac{\tilde{\delta}_{*}}{K+1} \mathbf{C}\right)-\frac{t \gamma_{*} \tilde{\delta}_{*}}{K+1}+ \\
\log \operatorname{det}\left(\frac{\gamma_{*}}{K+1} \tilde{\mathbf{C}}+\frac{K}{K+1} \mathbf{A}^{H}\left(\mathbf{I}+\frac{\tilde{\delta}_{*}}{K+1} \mathbf{C}\right)^{-1} \mathbf{A}\right)
\end{gathered}
$$

\section{DISCUSSION ON THE ACCURACY OF THE HIGH SNR APPROXIMANT.}

These results suggest to approximate $I\left(\sigma^{2}\right)$ at high SNR by $\bar{I}_{0}\left(\sigma^{2}\right)$ given by

$$
\bar{I}_{0}\left(\sigma^{2}\right)=t \log \frac{1}{\sigma^{2}}+\bar{L}(0)
$$

where $\bar{L}(0)$ is given by one of the expressions (15, (17), (24). It is of course important to verify that $\bar{I}_{0}\left(\sigma^{2}\right)$ is an accurate approximate of $I\left(\sigma^{2}\right)$ for realistic values of $r$ and $t$ at high SNR. In order to discuss on this point, we introduce the function $L\left(\sigma^{2}\right)$ defined by

$$
\begin{array}{rlc}
L\left(\sigma^{2}\right) & = & I\left(\sigma^{2}\right)-t \log \frac{1}{\sigma^{2}} \\
& =\mathbb{E}_{\boldsymbol{H}}\left[\log \operatorname{det}\left(\sigma^{2} \mathbf{I}_{t}+\mathbf{H}^{H} \mathbf{H}\right)\right]
\end{array}
$$

In particular, $L(0)=\mathbb{E}_{\boldsymbol{H}}\left(\log \operatorname{det} \mathbf{H}^{H} \mathbf{H}\right)$. The error $I\left(\sigma^{2}\right)-$ $\bar{I}_{0}\left(\sigma^{2}\right)$ can be written as

$$
I\left(\sigma^{2}\right)-\bar{I}_{0}\left(\sigma^{2}\right)=L\left(\sigma^{2}\right)-L(0)+L(0)-\bar{L}(0)
$$

This shows that the approximation error is due from one hand to $L\left(\sigma^{2}\right)-L(0)$, and the other hand on $L(0)-\bar{L}(0)$. We first discuss on the parameters that influence $L(0)-\bar{L}(0)$. We remark that Theorem 3.1 implies that

$$
L\left(\sigma^{2}\right)-\bar{L}\left(\sigma^{2}\right)=\epsilon\left(t, \sigma^{2}\right)
$$

where for each $\sigma^{2}>0, \epsilon\left(t, \sigma^{2}\right)$ converges towards 0 at rate $\frac{1}{t}$ (i.e. $\left|t \epsilon\left(t, \sigma^{2}\right)\right|$ is upperbounded by a function depending only on $\sigma^{2}$ ). Although (28) holds for each $\sigma^{2}>0$, it is not obvious that $L(0)-\bar{L}(0)$ still converges to 0 at rate $\frac{1}{t}$. It is even not clear whether $\frac{1}{t}(L(0)-\bar{L}(0))$ converges towards 0 . Indeed,

$$
\frac{1}{t}(L(0)-\bar{L}(0))=\frac{1}{t} \sum_{j=1}^{t} \log \lambda_{j}-\int_{\mathbb{R}^{+}} \log \lambda d \tilde{\mu}(\lambda)
$$

Function $\phi(\lambda)=\log \lambda$ is not lower bounded on $\mathbb{R}^{+}$. Therefore, (9) cannot be used without care. We also note that to establish Theorem 3.1, one uses very frequently that the norm of random matrix $\left(\mathbf{H}^{H} \mathbf{H}+\sigma^{2} \mathbf{I}_{t}\right)^{-1}$ is upper bounded by $\frac{1}{\sigma^{2}}$, a bound that is non random and independent of $t$ and $r$. This simple fact plays actually a very important role. If $\sigma^{2}=0$, this bound is equal to $+\infty$, thus showing that extending (28) to $\sigma^{2}=0$ needs the use of different technics and assumptions. This has been done in [1] devoted to the case $\mathbf{A}=0$ and $\tilde{\mathbf{C}}=0$. It is shown there that if the function $\phi$ is analytic in a neighbourood of the support of measure $\tilde{\mu}$, then, (9) holds, and

$$
\mathbb{E}_{\mathbf{H}}\left[\sum_{j=1}^{t} \phi\left(\lambda_{j}\right)-t \int_{\mathbb{R}^{+}} \phi(\lambda) d \tilde{\mu}(\lambda)\right]=o(1)
$$

Note that $o(1)$ can be replaced by $O\left(\frac{1}{t}\right)$ with some efforts. An important ingredient of [1] is that the probability that an eigenvalue of $\mathbf{H}^{H} \mathbf{H}$ lies outside the support of $\tilde{\mu}$ decreases to 0 faster than any term $\frac{1}{t^{p}}$ for each integer $p$. This means intuitively that it is possible to assume that the eigenvalues of $\mathbf{H}^{H} \mathbf{H}$ remain in the support of $\tilde{\mu} . \phi(\lambda)=\log \lambda$ satisfies the above assumption if the support of $\tilde{\mu}$ is included into an interval $(\epsilon,+\infty)$ where $\epsilon>0$. It is reasonable to conjecture that this kind of result can be extended to our context, and that $L(0)-\bar{L}(0)=O\left(\frac{1}{t}\right)$ if the support of $\tilde{\mu}$ is included into an interval $(\epsilon,+\infty)$. It is therefore important to study the support of $\tilde{\mu}$.

Proposition 5.1: If $r>t$ or $r=t$ and $K>K_{c}$ and $\mathbf{A}$ invertible, the support of $\tilde{\mu}$ is included in an interval $(\epsilon,+\infty)$ where $\epsilon>0$. Otherwise, 0 belongs to the support of $\tilde{\mu}$.

Sketch of proof. Due to the lack of space, we just show that if $r=t$ and $\mathbf{A}$ non invertible or $\mathbf{A}$ invertible, $K<K_{c}, 0$ belongs to the support of $\tilde{\mu}$. For this, we remark that the origin is a singular point of $\delta$ and $\tilde{\delta}$ (see Proposition 4.1). Therefore, 0 is also a singular point of $\tilde{\mathbf{T}}$ and of $\frac{1}{t} \operatorname{Tr} \tilde{\mathbf{T}}$. As $\frac{1}{t} \operatorname{Tr} \tilde{\mathbf{T}}$ is the Stieljès transform of $\tilde{\mu}, 0$ certainly belongs to the support of $\tilde{\mu}$; otherwise, it would be defined at $\sigma^{2}=0$.

Proposition 5.1 and the above discussion suggests $L(0)-\bar{L}(0)=O\left(\frac{1}{t}\right)$ if $r \neq t$ or $r=t, K>K_{c}, \mathbf{A}$ invertible, while its behaviour should be less favorable if $r=t$ and A non invertible or $\mathbf{A}$ invertible, $K<K_{c}$. In order to get some understanding of what can be expected in this last context, we consider a simple example for which explicit calculations can be done. Assume that $r=t$, and $\mathbf{H}$ is a zero mean i.i.d. matrix, i.e. that $\mathbf{C}=\tilde{\mathbf{C}}=\mathbf{I}_{t}$ and $K=0$. $L(0)=\mathbb{E}\left(\mathbf{H}^{H} \mathbf{H}\right)$ can be evaluated using the classical results of [3], and after some calculations it follows that $\bar{L}(0)=-t$ and that $L(0)-\bar{L}(0)$ remains bounded when $t \rightarrow+\infty$, but does not converge to 0 . This calculation suggests that for $r=t$ and $\mathbf{A}$ non invertible or $\mathbf{A}$ invertible and $K<K_{c}$, then $L\left(\sigma^{2}\right)-\bar{L}\left(\sigma^{2}\right)$ behaves quite differently according to whether $\sigma^{2} \neq 0$ or $\sigma^{2}=0$. In practice, this means that for a fixed value of $t$, then $L\left(\sigma^{2}\right)-\bar{L}\left(\sigma^{2}\right)$ should increase when $\sigma^{2}$ decreases. Figure 1 represents the relative error $\frac{L\left(\sigma^{2}\right)-\bar{L}\left(\sigma^{2}\right)}{L\left(\sigma^{2}\right)}$ in terms of $t$ for $S N R=+\infty, 30,20,10 d B$. In this experiment, $r=t, K<K_{c}$, the columns of $\mathbf{A}$ are normalized versions of directional vectors $a\left(\theta_{j}\right)=\left(1, e^{i \theta_{j}}, \ldots, e^{(t-1) \theta_{j}}\right)^{T}$ where the $\left(\theta_{j}\right)_{j=1, \ldots, t}$ are chosen uniformly on $[0,2 \pi]$ (A is invertible), while both transmit and receive antennas have exponential correlations with parameter $\rho_{t}=\rho_{r}=0.5$. Such a line of sight component is a reasonable model in the downlink of systems using macro diversity. The numerical evaluations confirm the above discussion. Figure 2 corresponds to the 
same experiments, but this time, $K>K_{c}$. As expected, the behaviour of $L\left(\sigma^{2}\right)-\bar{L}\left(\sigma^{2}\right)$ does not depend on $\sigma^{2}$, and for each $\sigma^{2}, \bar{L}\left(\sigma^{2}\right)$ appears to be a more accurate approximant of $L\left(\sigma^{2}\right)$ than if $K<K_{c}$.

We now briefly discuss on $L\left(\sigma^{2}\right)-L(0)$. Intuitively, the accuracy of the approximation $L\left(\sigma^{2}\right) \simeq L(0)$ depends on the distance between $\sigma^{2}$ and the smallest eigenvalues of $\mathbf{H}^{H} \mathbf{H}$. Therefore, better results are expected if 0 does not belong to the support of the deterministic equivalent of eigenvalue distribution of $\mathbf{H}^{H} \mathbf{H}$.

Figure 3 represents this time the relative error $\frac{I\left(\sigma^{2}\right)-\bar{I}_{0}\left(\sigma^{2}\right)}{I\left(\sigma^{2}\right)}$ in terms of $\frac{1}{\sigma^{2}}$. The parameters of the experiments are the same as below. 3 curves are represented: $r=t=4$ and $K<K_{c}, r=t=4$ and $K>K_{c}$, and $r=6$ and $t=4$. As expected, the relative error does not seem to converge to 0 in the first case. The convergence is very fast for $r=t=4$ and $K>K_{c}$, but slower if $r=1.5 t$, probably because the support of $\tilde{\mu}$ is closer from 0 in this case.

To conclude, the high SNR approximant $\bar{I}_{0}\left(\sigma^{2}\right)$ appears to be reliable for realistic values of $r$ and $t$ if $r=t, \mathbf{A}$ invertible $K>K_{c}$, and if $r \neq t$. It is however less accurate for $r=t$ and A non invertible or $r=t, \mathbf{A}$ invertible, $K<K_{c}$. Due to the lack of space, we do not discuss the engineering implications of these results. We just mention that if $r=t, \mathbf{A}$ invertible and $K>K_{c}$, then $I\left(\sigma^{2}\right)$ is very close to the capacity of the deterministic part of the channel. In particular, the Rayleigh component has no influence on the mutual information, a surprising result.

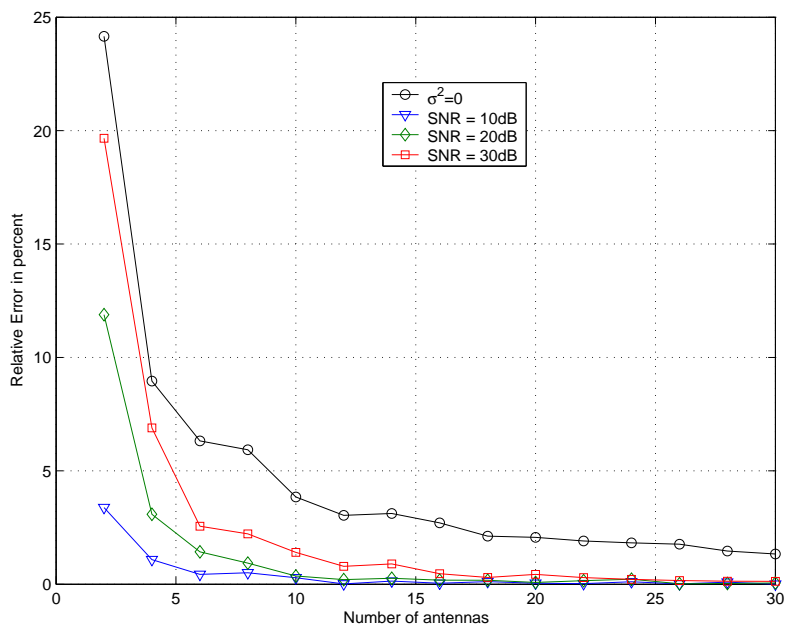

Fig. 1. Relative error for $r=t$ and $K<K_{c}$

\section{REFERENCES}

[1] Z. Bai, J. Silverstein, "CLT for linear statistics of large-dimensional sample covariance matrices", Ann. Probab., 32(1A):553-605, 2004.

[2] H. Cartan, "Théorie Elementaire des Fonctions Analytiques d'une ou Plusieurs Variables Complexes", Hermann, 1978.

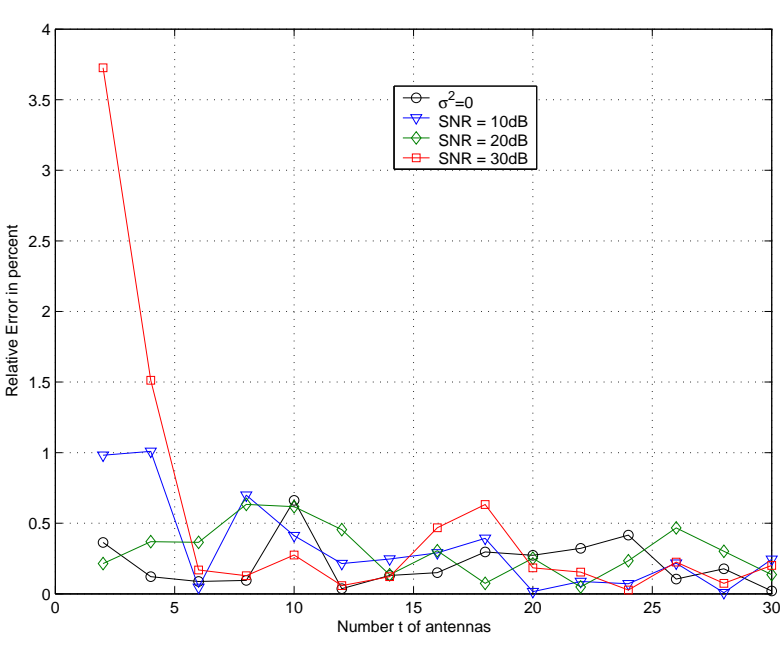

Fig. 2. Relative error for $r=t$ and $K>K_{c}$

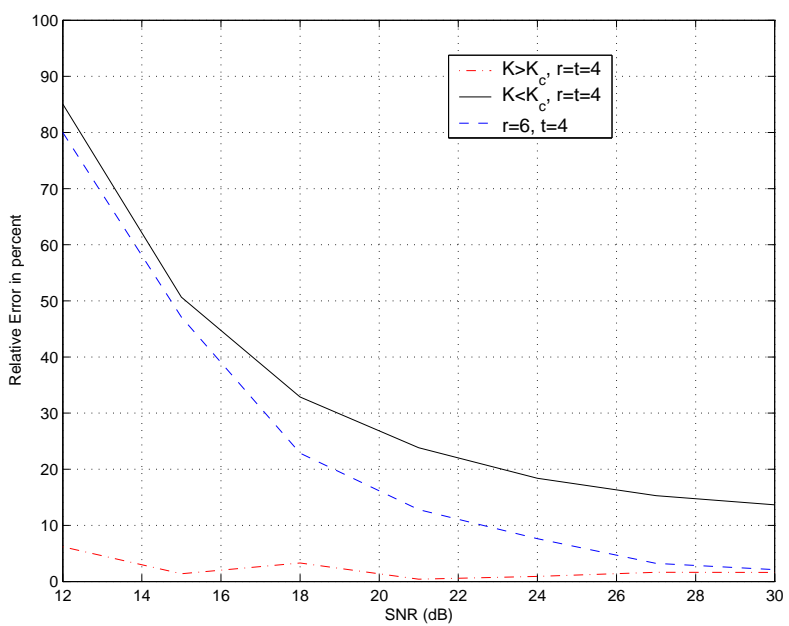

Fig. 3. Relevance of the high SNR approximations

[3] N.R. Goodman, "The distribution of the determinant of a complex Wishart distributed matrix", Annals of Mathematical Statistics, vol. 34, no. 1, pp. 178-180, March 1962.

[4] W. Hachem, Ph. Loubaton, J. Najim, "Deterministic equivalents for certain functionals of large random matrices", Preprint arXiv.math.PR/0507172v2, to appear in Annals of Applied Probabilty.

[5] M. Kang, M.S. Alouini, "Capacity of MIMO Rician channels", IEEE Trans. on Wireless Communications, vol. 5, no. 1, pp. 112-122, January 2006.

[6] M.G. Krein, A.A. Nudelman, "The Markov Moment Problem and Extremal Problems", American Mathematical Society, Providence, Rhode Island, 1997.

[7] G. Taricco, "On the capacity of separately correlated MIMO Rician channels", In Proc. Globecom 2006, Communication Theory Symposium, San Francisco, November 2006. 